Article

\title{
A Chalcone from Ashitaba (Angelica keiskei) Stimulates Myoblast Differentiation and Inhibits Dexamethasone-Induced Muscle Atrophy
}

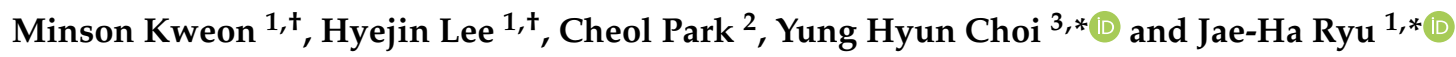 \\ 1 Research Institute of Pharmaceutical Sciences and College of Pharmacy, Sookmyung Women's University, \\ 100 Chungparo 47-Gil, Yongsan-Gu, Seoul 04310, Korea; minson-_-@nate.com (M.K.); \\ u9698115@naver.com (H.L.) \\ 2 Department of Molecular Biology, College of Natural Sciences, Dong-eui University, Busan 47340, Korea; \\ parkch@deu.ac.kr \\ 3 Department of Biochemistry, Dong-eui University College of Korean Medicine, Busan 47227, Korea \\ * Correspondence: choiyh@deu.ac.kr (Y.H.C.); ryuha@sookmyung.ac.kr (J.-H.R); \\ Tel.: +82-51-850-7413 (Y.H.C.); +82-2-710-9568 (J.-H.R.) \\ + Minson Kweon and Hyejin Lee contributed equally to this work.
}

Received: 20 September 2019; Accepted: 5 October 2019; Published: 10 October 2019

\begin{abstract}
Ashitaba, Angelica keiskei Koidzumi (AK), as a traditional medicine in Korea, Japan, and China, has been known as an elixir of life having therapeutic potential. However, there is no scientific evidence to support that Ashitaba can enhance or maintain muscle strength. To find a new therapeutic agent from the medicinal plant, we evaluated the anti-myopathy effect of chalcones from ethanol extract of AK (EAK) in cellular and animal models of muscle atrophy. To examine anti-myopathy activity, EAK was treated into dexamethasone injected rats and muscle thickness and histopathological images were analyzed. Oral administration of EAK ( 250 or $500 \mathrm{mg} / \mathrm{kg}$ ) alleviated muscle atrophic damages and down-regulated the mRNA levels of muscle-specific ubiquitin-E3 ligases. Among ten compounds isolated from EAK, 4-hydroxyderricin was the most effective principle in stimulating myogenesis of $\mathrm{C} 2 \mathrm{C} 12$ myoblasts via activation of p38 mitogen-activated protein kinase (MAPK). In three cellular muscle atrophy models with $\mathrm{C} 2 \mathrm{C} 12$ myoblasts damaged by dexamethasone or cancer cell-conditioned medium, 4-hydroxyderricin protected the myosin heavy chain (MHC) degradation through suppressing expressions of MAFbx, MuRF-1 and myostatin. These results suggest that the ethanol extract and its active principle, 4-hydroxyderricin from $\mathrm{AK}$, can overcome the muscle atrophy through double mechanisms of decreasing muscle protein degradation and activating myoblast differentiation.
\end{abstract}

Keywords: Ashitaba; Angelica keiskei; 4-hydroxyderricin; myogenesis; muscle atrophy

\section{Introduction}

With the increasing population of older individuals, incidence rates of various chronic diseases are also increasing. Chronic conditions and aging can lead to skeletal muscle wasting and weakness, i.e., muscle atrophy. Cachexia is a muscle wasting condition generated by chronic diseases, such as cancer, kidney failure, and diabetes. More than $30 \%$ of pancreatic cancer patients have died of muscle atrophy accompanied by resistance to chemotherapy and therapy-related side effects $[1,2]$. In addition, $50 \%$ of individuals over 80 years of age who suffer from fragility fractures have sarcopenia, an age-related muscle wasting condition [3]. Although the severity of muscle atrophy is increasing, only megestrol acetate has been approved by U.S. FDA for the treatment of cancer and AIDS induced cachexia. However, megestrol acetate has shown side effects such as insomnia, skin rash, impotence 
and fever. Thus, new agents having anti-myopathy potential with less side effects are needed to overcome the disease and age-associated muscle atrophy.

Muscle atrophy can be caused by impaired muscle regeneration following decreased number and dysfunction of muscle satellite cells (referred to as muscle stem cell) and elevated degradation of muscle protein [1]. Dexamethasone (a synthetic glucocorticoid) or conditioned media (CM) from cancer cell culture can lead muscle atrophy. They increase NF-kB-mediated expression of muscle specific E3 ligases such as muscle atrophy F-box (MAFbx) and muscle-specific RING finger protein-1 (MuRF1), which are associated with ubiquitin-proteasomal degradation of myofibers [4-6]. The dexamethasone-induced muscle atrophy in Sprague-Dawley rats was a well-established animal model to find therapeutic agents for muscle atrophy under several wasting conditions [7].

Angelica keiskei Koidzumi (AK) (Japanese name "Ashitaba" meaning tomorrow leaf, Korean name "Shinsuncho" meaning elixir of life, Umbelliferae) has been used as a traditional medicine [8] and diverse dietary supplements of Ashitaba tea or juice were prepared. The inhabitants of Hachijo-jima, a village famous for longevity in Japan, believe that Ashitaba has been improving their health [9]. Roots and leaves of Ashitaba were known to be effective for improving asthma, chronic hepatitis, diabetes, gastritis, high blood pressure, obesity, and psoriasis. In addition, Ashitaba has been transmitted in folk remedy in Asian countries as a treatment for muscle and joint pain.

As chalcones of Ashitaba, including 4-hydroxyderricin (4-HD), isobavachalcone, xanthokeismin $\mathrm{A}$, and xanthoangelol $\mathrm{B}, \mathrm{E}, \mathrm{D}$, and $\mathrm{F}$ have been reported to possess a wide range of pharmacological activities including anti-oxidative and anti-inflammatory potential. Especially, two chalcones, 4-HD and xanthoangelol have attracted attention to develop herbal supplements or medicines due to their pharmacological potentials and high contents in this edible herb [10,11]. Recently, metabolomic and lipidemic analyses of human plasma revealed that five components of AK including 4-HD are responsible for preventive effects against liver diseases, type 2 diabetes, obesity and atherosclerosis [12]. Studies on toxicity and metabolism of 4-HD and xanthoangelol were published in rat or human models. These reports encourage their pharmaceutical applications $[13,14]$. The regulatory effect of two chalcones on glucose metabolism in muscle [15] implies their potential application for muscle strength, regarding the beneficial relationship between muscle hypertrophy and glucose metabolism [16].

In this study, we identified a chalcone compound, 4-HD as the most potent myogenesis-stimulating agent among compounds purified from AK. We investigated the preventive and protective effects of AK extract and 4-HD against muscle atrophy both in vivo and in vitro models and disclosed underlying action mechanisms.

\section{Materials and Methods}

\subsection{Animals}

Sprague-Dawley rats (6 weeks old, male) were purchased from Samtako (Osan, Korea). They were maintained in controlled environment $\left(23 \pm 1{ }^{\circ} \mathrm{C}, 55 \pm 5 \%\right.$ relative humidity) under a $12 \mathrm{~h}$ light/dark cycle for acclimation. Rats were group housed in poly-propylene cages ( 3 rats per cage) and were provided ad libitum access to water and a standard laboratory diet. All animal experiments were conducted according to the National Institutes of Health Guide for the Care and Use of Laboratory Animals (8th edition, revised in 2011) and approved by the Institutional Animal Committee of Dong-eui University (\#A2017-006/2017).

\subsection{Experimental Procedures}

After 8 days acclimation, these rats (mean weight was $253 \mathrm{~g}$ ) were randomly divided into five groups: (i) the intact vehicle control group, (ii) dexamethasone (Dex) control group, (iii) Dex and ethanol extract of A. keiskei (EAK) $250 \mathrm{mg} / \mathrm{kg}$-treated group, (iv) Dex and EAK $500 \mathrm{mg} / \mathrm{kg}$-treated group, and (v) Dex and oxymetholone-treated group (6 animals per group). Different groups were randomly treated in each experiment. To induce muscle atrophy, Dex (1 mg/kg body mass) was intraperitoneally 
injected daily for 7 days. Two different concentrations of EAK ( 250 and $500 \mathrm{mg} / \mathrm{kg}$ body mass) were orally administered once daily for 28 days. Oxymetholone ( $50 \mathrm{mg} / \mathrm{kg}$ body mass) was administered orally for the same period as EAK administration. Oxymetholone is a synthetic anabolic steroid used as an agent to accelerate muscle growth and shows ameliorative effects on muscle wasting after dexamethasone treatment in animal models [17].

\subsection{Measurement of Body Weight and Gastrocnemius Muscle Thickness}

Body weight was measured at the end of test material administration using an automatic electronic balance machine (Precisa Instruments, Dietikon, Switzerland). A $25 \mathrm{mg} / \mathrm{kg}$ of zoletil (Zoletil $50^{\circledR}$; Virbac, Nice, France) was injected into the abdominal cavity of each rat at the end of test material administration and the gastrocnemius muscle (GA) was exposed. The thickness of exposed GA of the left hind limb was measured using an electronic digital caliper (Mytutoyo, Tokyo, Japan) and photographed.

\subsection{Histopathological Analysis}

Samples of gastrocnemius muscle were separated, fixed in 10\% neutral-buffered formalin for $24 \mathrm{~h}$, embedded in paraffin, transversely sectioned ( $3 \mu \mathrm{m}$ in thickness), and then stained with hematoxylin and eosin (H\&E) for general histopathological analysis. Histopathological profiles of each sample were then determined by light microscopy observation (Nikon, Tokyo, Japan).

\subsection{Preparation of Chalcones from Roots of $A K$}

Dried roots of AK were purchased from Sengdong-nongsan Co. (Chungju, Korea) and authenticated by Prof. Kisook Yang at the College of Pharmacy, Sookmyung Women's University. A voucher specimen (No. SPH 1501) was deposited at the Herbarium of Sookmyung Women's University. Chalcones were isolated from ethanol extract $(26 \mathrm{~g})$ of EAK as previously reported and dissolved in dimethyl sulfoxide for treating cells [10]. All chemical structures were elucidated by spectroscopic data analysis followed by comparison with previously described data $[10,18]$, and the purities $(>96 \%)$ were determined by ${ }^{1} \mathrm{H}-\mathrm{NMR}$ spectra and HPLC. The HPLC analysis was performed using Waters 1525 system (Milford, MA, USA). A reverse phase column (ODS-2, $5 \mu \mathrm{m}, 150 \times 4.6 \mathrm{~mm}$ i.d., GL Sciences Inc., Tokyo, Japan) was eluted with $80 \%$ methanol (flow rate $1 \mathrm{~mL} / \mathrm{min}$ ), and monitored with PDA detector at $360 \mathrm{~nm}$. Copies of the original spectra are obtainable from the corresponding author.

\subsection{Cell Culture, Myoblast Differentiation and Preparation of Conditioned Medium of CT26 Cancer Cells}

C2C12 myoblast cells obtained from the American Type Culture Collection (ATCC, Manassas, VA, USA) were differentiated for 3 days as demonstrated in our previous studies [19]. We prepared CT26 murine colon carcinoma cell-conditioned medium (CM) according to previous studies [19]. To prepare 4-HD-treated CM (HD-CM), CT26 cells were plated and treated with 4-HD for $24 \mathrm{~h}$ in DMEM containing $10 \%$ FBS. After washing with PBS, cells were incubated in serum free DMEM for another $24 \mathrm{~h}$. The prepared CM was diluted with fresh differentiation medium (DMEM containing $2 \%$ horse serum) to make a final concentration of $30 \%$ for treating myoblasts or myotubes.

\subsection{MyoD-Reporter Gene Assay}

To measure MyoD transcriptional activity, C2C12 cells were transiently transfected with MyoD-responsive reporter 4RTK-luciferase (RTK-Luc) and pBP-MyoD constructs using Lipofectamine ${ }^{\circledR}$ 2000 Reagent (Invitrogen, Carlsbad, CA, USA) [19]. At $24 \mathrm{~h}$ post transfection, cells were treated with test compounds for $24 \mathrm{~h}$. Luciferase assay was then performed using cell lysates and a commercial luciferase assay kit (Promega, Madison, WI, USA). Data are reported as relative luciferase activity (RLU) divided by $\beta$-galactosidase activity. 


\subsection{Immunostaining of Myosin Heavy Chain (MHC)}

In brief, treated myoblasts or myotubes were fixed, permeabilized, and incubated with a primary antibody against MHC (MAB4470, R\&D Systems, Minneapolis, MN, USA) at $4{ }^{\circ} \mathrm{C}$ overnight followed by incubation with a goat anti-mouse antibody conjugated with Alexa Fluor 568 (Life Technologies, Carlsbad, CA, USA) [20]. These cells were counterstained with DAPI (4' ${ }^{\prime}, 6$-diamidino-2-phenylindole, Sigma-Aldrich). MHC immunofluorescence was then detected under a fluorescence microscope (Olympus, Tokyo, Japan). A red fluorescence indicates MHC expression while multinucleated myotubes are observed with DAPI (blue-colored) counterstaining. The number of MHC-expressing multinucleated (containing more than four nuclei) myotubes in the counting field was presented as a relative change to that of control group. Images were captured with a Nikon ECLIPSE TE-2000U microscope and processed with NIS-Elements F software (Nikon) and Photoshop CS5 (Adobe).

\subsection{Western Blot Analysis}

$\mathrm{C} 2 \mathrm{C} 12$ cells were differentiated in DM with the test sample and cell lysates were subjected to Western blotting analysis to determine protein expression of myogenic markers and E3 ligases. Harvested cells were suspended in lysis buffer ( $50 \mathrm{mM}$ Tris, $\mathrm{pH} 7.4,150 \mathrm{mM} \mathrm{NaCl}, 10 \%$ glycerol, $1.5 \mathrm{mM}$ $\mathrm{MgCl}_{2}, 1 \mathrm{mM}$ EGTA, $1 \%$ Triton X-100, $10 \mathrm{mM} \mathrm{NaF}, 1 \mathrm{mM} \mathrm{Na}_{3} \mathrm{VO}_{4}$, and protease inhibitor cocktail) and proteins were loaded onto sodium dodecyl sulfate-polyacrylamide gel, electrophoresed, and transferred to polyvinylfluoride membrane in transfer buffer ( $39 \mathrm{mM}$ glycine, $48 \mathrm{mM}$ Tris base, $0.037 \%$ SDS, and $20 \%$ methanol) for $2 \mathrm{~h}$. The membrane was then incubated with antibodies specific to MHC (Santa Cruz Biotechnology, Dallas, TX, USA), MyoD (Santa Cruz Biotechnology), Myogenin (Santa Cruz Biotechnology), MAFbx (Santa Cruz Biotechnology), and MuRF1 (Santa Cruz Biotechnology). p38 MAPK activation was analyzed by using antibodies against phospho-p38 (Cell Signaling Technology, Danvers, MA, USA) and p38 MAPK (Cell Signaling Technology). Pan-cadherin (Sigma, St. Louis, MO, USA) was used as a loading control.

\subsection{RNA Extraction and Reverse Transcription Polymerase Chain Reaction (RT-PCR)}

Gene expression of E3 ligases was analyzed by RT-PCR. RNAs were extracted from myoblasts or myotubes using Trizol Reagent (Life Technologies). First-strand cDNA synthesis was then performed using Labopass ${ }^{\mathrm{TM}} \mathrm{CDNA}$ synthesis kit (Cosmogenetech, Seoul, Korea) according to the manufacturer's recommendation. PCR was carried out as follows: $5 \mathrm{~min}$ at $94{ }^{\circ} \mathrm{C}, 30$ cycles of $94{ }^{\circ} \mathrm{C}$ for $1 \mathrm{~min}, 56{ }^{\circ} \mathrm{C}$ for $1 \mathrm{~min}$, and $72{ }^{\circ} \mathrm{C}$ for $1 \mathrm{~min}$, and a 5-min incubation at $72{ }^{\circ} \mathrm{C}$. All mRNA levels were normalized to glyceraldehyde 3-phosphate dehydrogenase mRNA levels. The final PCR amplicons were then separated using electrophoresis with a $1.8 \%$ agarose gel. The primers used for the amplifications are shown in Table 1.

Table 1. Oligonucleotide primer sequences used for the RT-PCR analysis.

\begin{tabular}{cccc}
\hline Gene Name & Forward Primer & Reverse Primer & Accession Number \\
\hline$M A F b x$ & CGACCTGCCTGTGTGCTTAC & CTTGCGAATCTGCCTCTCTG & BC027211 \\
$M u R F 1$ & GGTGCCTACTTGCTCCTTGT & CTGGTGGCTATTCTCCTTGG & NC_000070 \\
GAPDH & TGCACCACCAACTGCTTAG & GGCATGGACTGTGGTCATGAG & BC0960 42 \\
\hline
\end{tabular}

$M A F b x$, muscle atrophy F-box (MAFbx/atrogin-1); MuRF1, muscle RING finger protein-1; GAPDH, glyceraldehyde 3-phosphate dehydrogenase.

\subsection{Statistical Analysis}

All values are presented as mean \pm standard deviation (SD). Differences were assessed using Student's $t$-test or one-way analysis of variance (ANOVA) followed by Duncan's test. All experiments were performed at least three times. Difference with a $p$ value of less than 0.05 was considered statistically significant. 


\section{Results}

\subsection{Ethanol Extract of Angelica keiskei Alleviates Dexamethasone-Induced Muscle Atrophy in Rats}

To investigate the protective effect of ethanol extract of Angelica keiskei Koidzumi (EAK) against muscle atrophy induced by dexamethasone in rats, we measured body weight and gastrocnemius muscle thickness. As shown in Figure 1A,B, dexamethasone treatment decreased body weight and gastrocnemius muscle thickness compared with vehicle control. However, EAK treatment $(500 \mathrm{mg} / \mathrm{kg})$ significantly recovered the damage. As shown in histopathological staining of muscle (Figure 1C), dexamethasone increased thickness of perimysium and endomysium, and decreased muscle fiber diameters compared with the intact vehicle control. EAK ameliorated these atrophic damages in a dose-dependent manner. Next, we investigated the mechanism involved in EAK-mediated muscle protection in animal by measuring mRNA levels of muscle-specific E3 ligases. Dexamethasone significantly increased expressions of muscle RING finger protein-1 (MuRF1) and muscle atrophy $F$ box (MAFbx/atrogin-1) in gastrocnemius muscles (Figure 1D). However, EAK (500 mg/kg) decreased these expression levels of MuRF1 or MAFbx mRNA, while it increased expression levels of MyoD and myogenin (myogenic factors). Oxymetholone, a representative $17 \alpha$-alkylated anabolic-androgenic steroid, was known to inhibit the glucocorticoid-induced loss of body weight and muscle via its anabolic effects [17]. We used oxymetholone as a positive control in animal study (50 mg/kg).

A

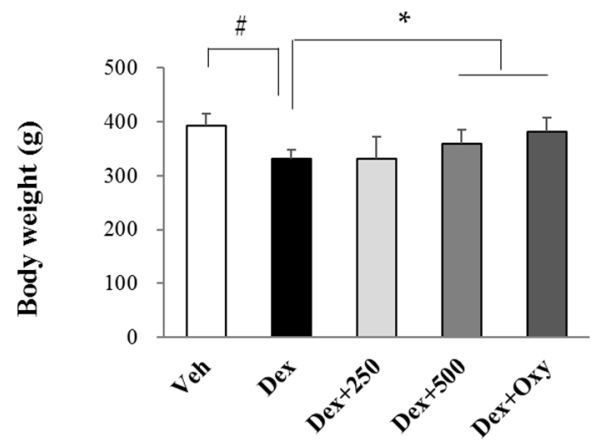

C

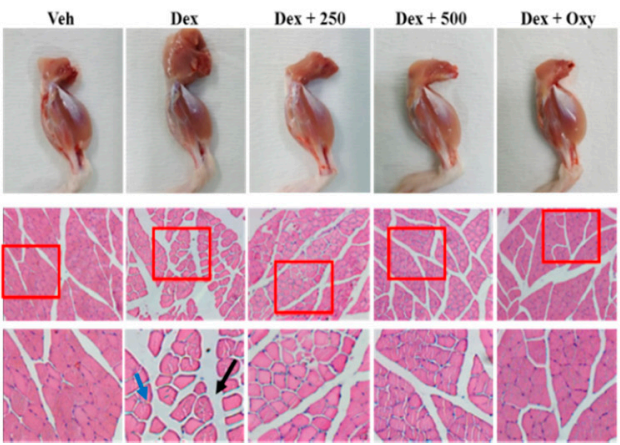

B

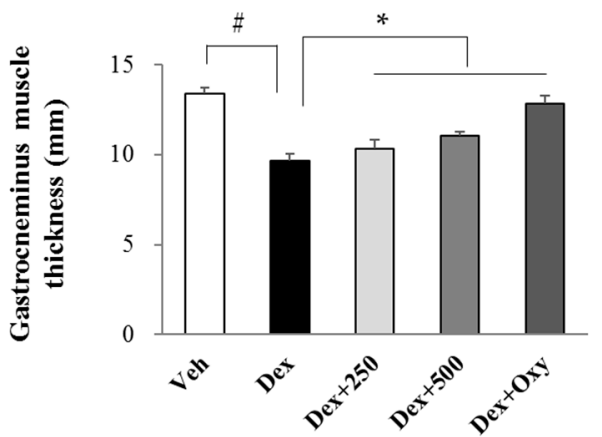

D

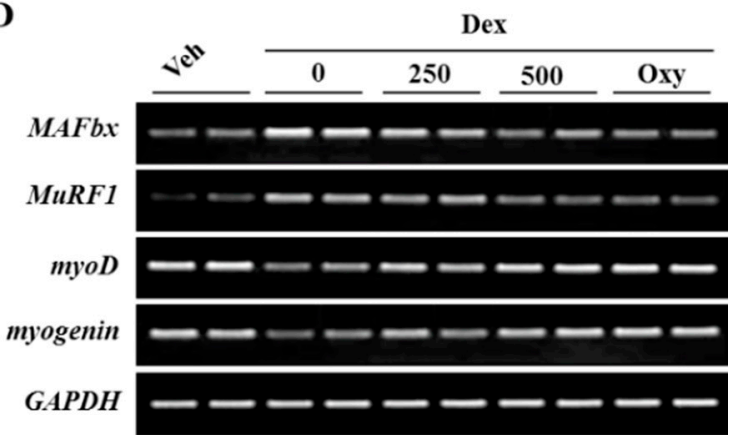

Figure 1. Anti-myopathy activity of ethanol extract of Angelica keiskei in dexamethasone-induced muscle atrophied rats. Effects of ethanol extract of AK (EAK) (250 and $500 \mathrm{mg} / \mathrm{kg}$ ) on body weight (A) and gastrocnemius muscle thickness (B). (C) Gross appearance and histopathological images of the hind limb of rats. Black arrow indicates the perimysium and blue arrow indicates the endomysium. (D) Changes in gastrocnemius muscle mRNA expression were measured by reverse transcription polymerase chain reaction (RT-PCR). Oxymetholone (Oxy) was used as a positive control (50 mg/kg). Data are expressed as mean $+\mathrm{SD}(\mathrm{n}=6)$. \# $p<0.005$ vs. vehicle control rat $($ Veh $) ;{ }^{*} p<0.005$ vs. dexamethasone treated rat (Dex). 


\subsection{EAK and Its Components Promote Myogenesis}

Mouse skeletal myoblast C2C12 is derived from mouse satellite cell and well-established cell line to study the myogenic potential of chemicals [21]. C2C12 myoblasts were differentiated with EAK (10, 100, or $1000 \mathrm{ng} / \mathrm{mL}$ ), which dose-dependently enhanced expression levels of MHC (Figure 2A,B) as a marker of terminal myogenesis [19]. We observed the cylinder-shaped multinucleated myotubes (containing more than four nuclei and indicating mature myotubes) by immunostaining for MHC and DAPI [19]. The number of multinucleated myotubes was increased by EAK in a dose-dependent manner. We also evaluated myogenic activities of EAK by measuring MyoD transcriptional activities using a reporter gene assay. EAK dose-dependently increased MyoD transcriptional activities (Figure 2C).

A

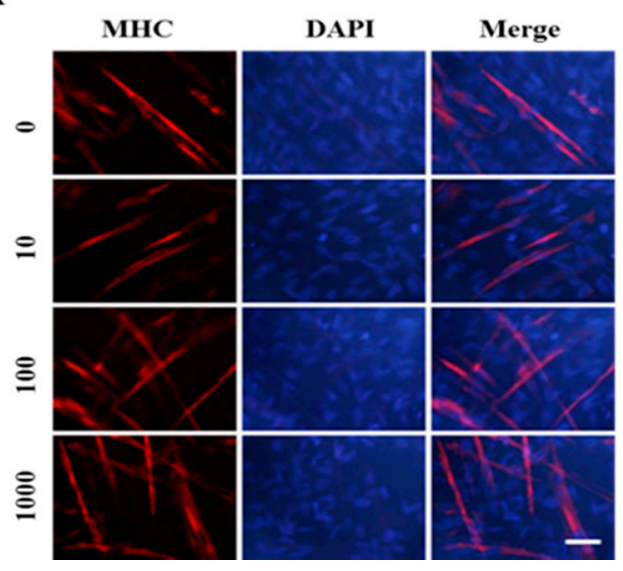

D

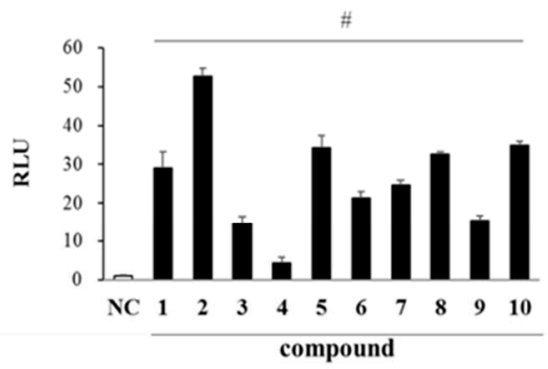

B

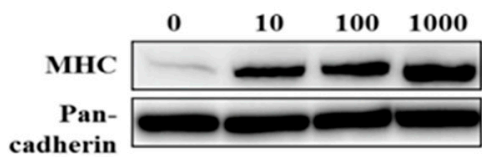

C

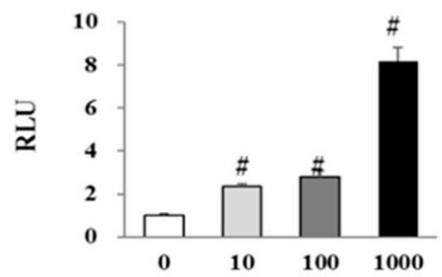

$\mathbf{E}$

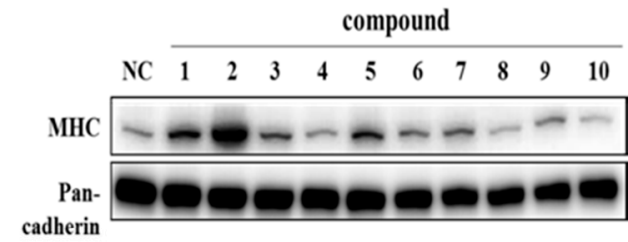

F<smiles>COc1ccc(C(=O)/C=C/c2ccc(O)cc2)c(O)c1CC=C(C)C</smiles>

Figure 2. Effects of EAK and AK compounds on myogenesis of myoblasts. (A) C2C12 cells were differentiated in differentiation medium (DM) supplemented with EAK $(10,100$, or 1000 $\mathrm{ng} / \mathrm{mL}$ ) for 3 days and immunostained with antibody to myosin heavy chain (MHC, red) and 4',6-diamidino-2-phenylindole (DAPI, blue). Scale bar $=200 \mu \mathrm{m}$. (B) Expression level of MHC was determined by Western blotting analysis. (C) C2C12 cells were transiently transfected with 4RTK-luciferase and MyoD-pBP plasmid, and then treated with EAK as described in materials and methods. MyoD transactivation is presented as relative luciferase activity (RLU). Data are expressed as mean \pm standard deviation (SD). \# $p<0.001$ vs. $0 \mathrm{ng} / \mathrm{mL}$ of EAK. (D) C2C12 cells were transiently transfected with 4RTK-luciferase and MyoD-pBP plasmid, and then treated with AK compounds 1-10 $(100 \mathrm{pM})$ for an additional $24 \mathrm{~h}$. Data are expressed as mean \pm standard deviation (SD). NC, negative control (DMSO); 1-10, compounds 1-10. \# $p<0.001$ vs. NC. (E) C2C12 cells were differentiated in the presence of each AK compounds 1-10 and then expression level of MHC was determined by Western blotting analysis. (F) Chemical structure of 4-HD. 
EAK was partitioned with ethyl acetate and water to isolate myogenic principles. Ethyl acetate-soluble fractions were subjected to silica gel column chromatography from which ten chalcones were purified: isobavachalcone (1), 4-hydroxyderricin (4-HD) (2), xanthoangelol E (3), xanthoangelol D (4), xanthoangelol (5), xanthoangelol F (6), xanthokeismin A (7), 1-[2,4-dihydroxy-3-(6,7-dihydroxy-3, 7-dimethyl-2-oxtenyl)phenyl] 3-(4-hydroxyphenyd, 1-2-prepen-1-one chalcone (8), 1-[2-hydroxy-3(7-hydroxy-3,7-dimethyl-2,5-ocadienyl)-4-methoxyphenyl]-3-(4-hydroxyphenyl)-2-propen-1-onechalcone (9), and xanthoangelol B (10). Their chemical structures were identified by spectroscopic analysis and comparison with previous data $[18,22]$.

All AK compounds increased MyoD transcriptional activity (Figure 2D) and myosin heavy chain (MHC) expressions in $100 \mathrm{pM}$ concentrations (Figure 2E). The effective dose of compounds was unexpectedly very low. With considering the effective dose in our animal study $(500 \mathrm{mg} / \mathrm{kg}$ ), the in vitro concentration could not be extrapolated to effective dose in in vivo study. Since 4-HD (compound 2, Figure 2F) showed the strongest activity among them in MyoD transactivation and MHC expression, we used this compound in further study. The main constituents of EAK were identified as 4-HD and xanthoangelol (compound 5) by HPLC, and their contents were quantified as $2.8 \%$ and $4.8 \%$ $(\mathrm{w} / \mathrm{w})$ respectively (data not shown).

\subsection{4-HD Stimulates Myogenesis}

To examine the myogenic effect of 4-HD, $\mathrm{C} 2 \mathrm{C} 12$ myoblasts were differentiated in the presence of 4-HD. As shown in Figure 3, 4-HD dose-dependently enhanced expression of MHC and the number of multinucleated myotubes in sub-nanomolar concentrations. These data demonstrate that picomolar concentration of 4-HD is sufficient to induce myoblast differentiation from $\mathrm{C} 2 \mathrm{C} 12$ myoblasts.

A

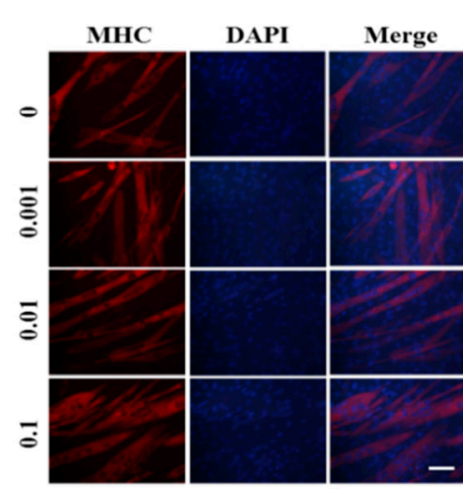

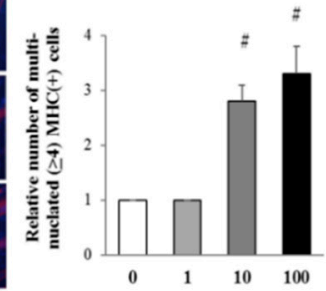

B

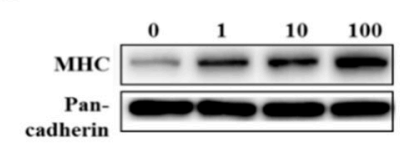

C

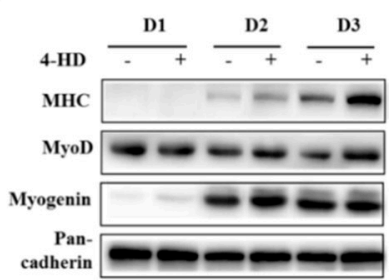

Figure 3. Effect of 4-HD on myogenesis of myoblasts. (A) Differentiated C2C12 cells in the presence of 4-HD (1, 10, $100 \mathrm{pM})$ were immunostained with antibody to MHC (red) and 4',6-diamidino-2-phenylindole (DAPI, blue). Multinucleated ( $>4$ nuclei) and MHC-expressing myotubes were counted from randomly selected areas. Scale bar $=200 \mu \mathrm{m}$. \# $p<0.005$ vs. $0 \mathrm{nM}$. (B) Expression levels of MHC were determined by Western blotting analysis. (C) C2C12 myoblasts were treated with 4-HD (100 pM) during myogenesis period (D1, D2, D3) and levels of myogenic factors were measured by Western blotting analysis.

Expression levels of MHC and myogenin were gradually increased during differentiation period (Figure 3C), and were further increased by 4-HD treatment (100 pM). The expression level of MyoD, as an initiator of myogenesis, was gradually decreased after DM treatment. However, 4-HD treatment maintained MyoD level during myogenesis compared with the respective control myoblasts. 


\subsection{4-HD Stimulates Myogenesis by p38 MAPK Activation}

To determine the mechanism involved in the myogenic activity of 4-HD, we examined activation of p38 mitogen-activated protein kinase (MAPK) that was reported to activate myoblast differentiation [23]. As shown in Figure 4A, basal level of phospho-p38 MAPK was gradually increased to drive myogenesis. 4-HD further increased the level of phospho-p38 MAPK during myogenesis. To clarify the role of p38 MAPK in 4-HD-induced myogenesis, C2C12 myoblasts were pretreated with $10 \mu \mathrm{m}$ of SB203580, a pharmacological inhibitor of p38 MAPK, ahead of 4-HD. SB203580 reduced basal expression of MHC and also suppressed 4-HD induced-MHC expression to basal level (Figure 4B).

A

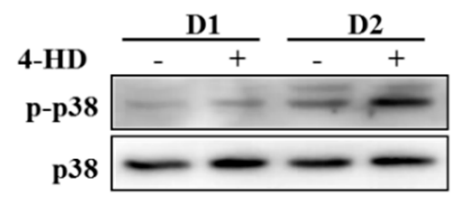

B

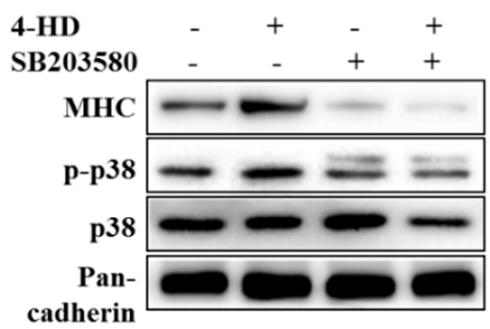

Figure 4. Effect of 4-HD on p38 MAPK activation during myogenesis. (A) 4-HD (100 pM) treated myoblasts were harvested at indicated differentiation periods. (B) Confluent myoblasts were pre-incubated with SB203580 $(10 \mu \mathrm{m})$ for $1 \mathrm{~h}$, followed by 4-HD stimulation for 2 days. The phospho-p38 MAPK was measured by Western blotting analysis.

\subsection{4-HD Protects Against Muscle Wasting In vitro}

To evaluate a protective effect of 4-HD, we adopted three types of muscle wasting models by using dexamethasone or conditioned media (CM) of colon cancer cells. Firstly, $\mathrm{C} 2 \mathrm{C} 12$ myoblasts were differentiated with DM containing dexamethasone $(20 \mu \mathrm{M})$ in the presence or absence of 4-HD for 3 days. Dexamethasone, a synthetic glucocorticoid, was reported to induce muscle atrophy in vitro and in vivo $[17,24]$. Dexamethasone significantly decreased MHC expression and multinucleated myotubes, and enhanced MAFbx expression, while 4-HD (100 pM) decreased the dexamethasone-induced MHC loss and MAFbx expression. The level of MuRF1 was significantly reduced by 4-HD treatment compared with dexamethasone group, even though it was not significantly enhanced by dexamethasone treatment (Figure 5).
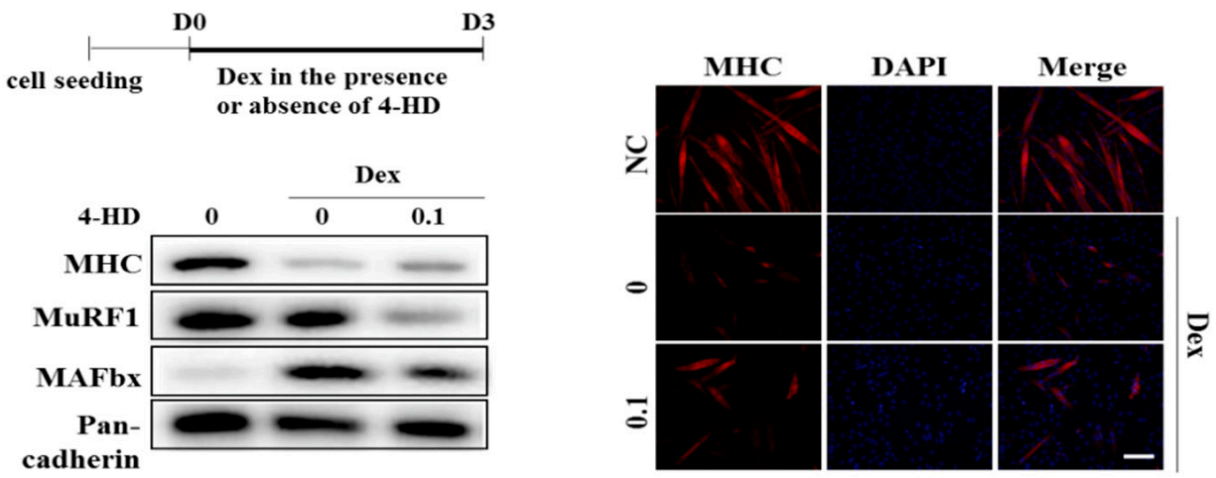

Figure 5. Protective effect of 4-HD against muscle wasting in vitro. $\mathrm{C} 2 \mathrm{C} 12$ Cells were treated according to the scheme and expression levels of MHC and muscle E3 ligases were determined by Western blotting analysis. MHC and DAPI were immunostained in myotubes. Scale bar $=200 \mu \mathrm{m}$.

Secondly, differentiation of $\mathrm{C} 2 \mathrm{C} 12$ myoblasts were induced into myotubes for 3 days with $30 \%$ conditioned media (CM) or 4-HD (0.1 or $1 \mathrm{nM})$ treated CM (HD-CM) (Figure 6A). As expected, CM 
decreased MHC and increased myostatin protein levels compared with DM control. However, 30\% HD-CM reversed levels of MHC and myostatin that might come from the decreased release of atrophic factors from cancer cells by 4-HD treatment. CM increased mRNA levels of $M A F b x$ compared with DM control whereas HD-CM blunted MAFbx expression. However, mRNA expression of MuRF1, another E3 ligase, was not affected by 4-HD treatment.

A
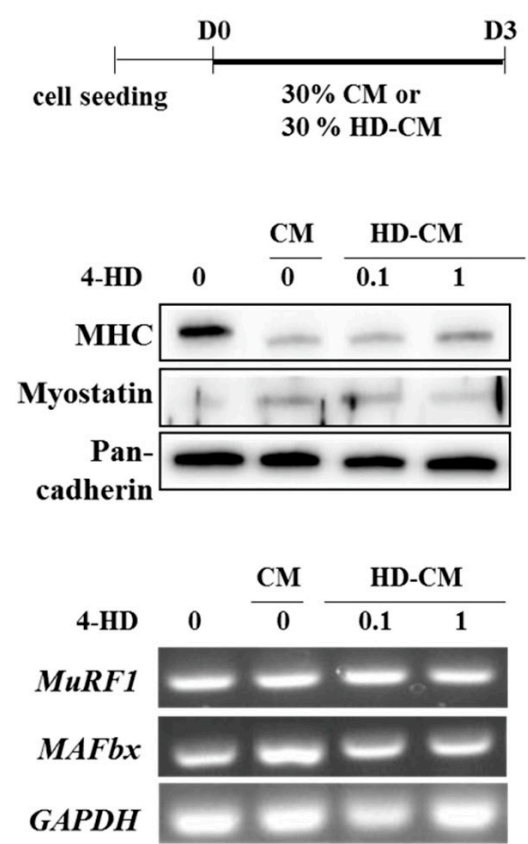

B
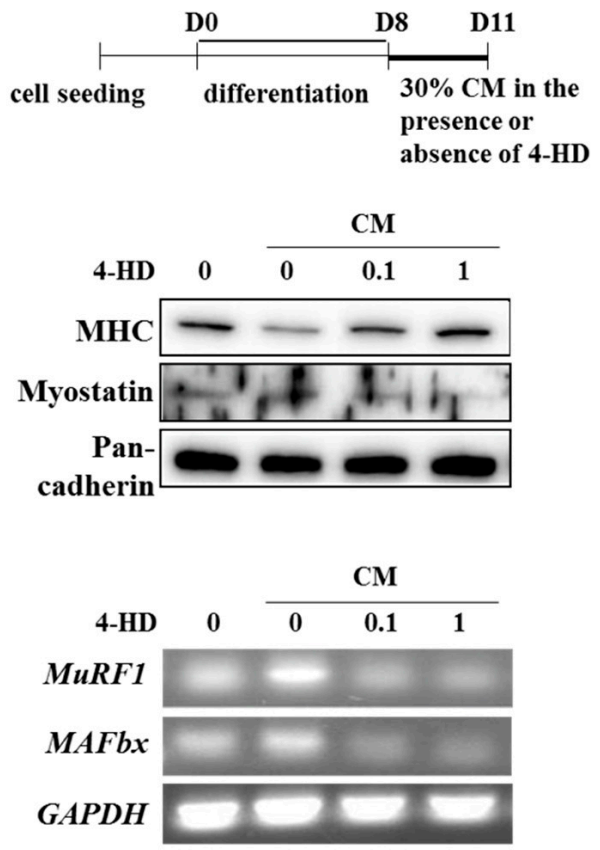

Figure 6. Preventive and protective effects of 4-HD against muscle wasting in vitro. Myoblasts (A) and myotubes (B) were treated according to the scheme shown, and expression levels of MHC, myostatin, and muscle E3 ligases were determined by Western blotting analysis or RT-PCR.

Thirdly, myoblasts were differentiated for 8 days followed by incubation with $30 \% \mathrm{CM}$ to induce myotube damage. CM significantly decreased MHC, increased myostatin protein, and increased mRNA expression of $\mathrm{E} 3$ ligases in myotubes. However, 4-HD reversed such effects of $\mathrm{CM}$ in a dose-dependent manner. 4-HD protected against CM-induced MHC degradation by suppressing expression of E3 ligases (MAFbx and MuRF1) and myostatin compared with CM control (Figure 6B). These results suggest that 4-HD may protect muscle wasting in cancer-induced cachexia condition.

\section{Discussion}

Ashitaba, Angelica keiskei Koidzumi (AK), has been used as both a vegetable and folk medicine in Asian countries. Recently many dietary supplements were developed using Ashitaba for heartburn, stomach ulcers, high blood pressure, high cholesterol, gout, and constipation. Ethanol extracts of AK and the main ingredients (4-hydroxyderricin and xanthoangelol) were reported to regulate glucose uptake and improve insulin sensitivity in muscle in vivo or in vitro models [25]. Considering the positive effects of AK extract and main ingredients on glucose metabolism in skeletal muscle, we hypothesized that AK could improve physical activities as well as muscle strength. In our preliminary study, EAK $(250,500 \mathrm{mg} / \mathrm{kg}$ ) significantly extended running time of mice in treadmill test as compared with dexamethasone-damaged groups (Figure S1). So, we tried to find out the active constituent of EAK and disclose the underlying mechanism of action.

Muscle quiescent satellite cells can be activated by exercise or muscle injury to regenerate and repair injured muscles. Activated myogenic satellite cells undergo myogenesis to become 
myoblasts (proliferating satellite cells), and then differentiated into mature myotubes. During myoblast differentiation, various myogenic factors such as $\mathrm{MyoD}$, myogenic factor (Myf)-5, myogenin, myogenic regulatory factor (MRF)-4, and myosin heavy chain (MHC), not only contribute to myogenic lineage specification of muscle stem cells, but also initiate and terminate myoblast differentiation [26,27].

Considering the role of satellite cells, we purified ten chalcones from AK, which has been used as food and herbal medicine, and we identified activators of satellite cells as potential therapeutic agents for muscle atrophy. Among tested chalcones, 4-hydroxyderricin (4-HD) was the most potent myogenic constituent of AK. At the same concentration $(10 \mathrm{ng} / \mathrm{mL}), 4-\mathrm{HD}$ showed a 15-fold transcriptional activation of MyoD compared with EAK. In the literature, 4-HD has been reported to exhibit anti-inflammatory, anti-microbial, anti-platelet, anti-diabetic, and anti-obesity activities [11,28]. The present study demonstrated that 4-HD stimulates myogenesis in dose- and time-dependent manners via p38 mitogen-activated protein kinase (MAPK) activation. The p38 MAPK phosphorylates E proteins, SWI/SNF subunit BAF60, or Mef2 to form complex with MyoD which in turn initiate the expression of myogenic factors [29]. As p38 MPAK activation is an essential mechanism for myogenesis [23], we observed activation of p38 MAPK by 4-HD during myogenesis, while SB203580 (a p38 MAPK inhibitor) abrogated the myogenic effects of 4-HD, implying the central role of p38 MAPK in 4-HD-induced myogenesis. In this study, we report for the first time the myogenic potential of 4-HD and AK that can be applied to treatment of muscle atrophy.

Another considerable therapeutic strategy for muscle atrophy is inhibition of muscle protein degradation. Under cachexic conditions, myo-proteins undergo ubiquitin modification by E3 ubiquitin ligases and proteasome-dependent degradation [5,6]. Thus, increased expression of E3 ubiquitin ligases indicate the progress of muscle atrophy accompanied by myofibrillar and intracellular proteins breakdown $[17,30]$. Bodine et al. have shown that MAFbx or MuRF1-deficient mouse can overcome muscle atrophy, whereas MAFbx overexpressing myoblasts exhibit retardation in myotube formation [31]. Although inhibitors of proteasomal degradation such as bortezomib and MG132 can attenuate myo-protein degradation, their application for treating muscle diseases has not been reported yet.

Myostatin, a negative regulator of skeletal muscle growth, contributes to the loss of muscle mass associated with aging and muscle diseases. In a recent study, knockouts (Mst-/-) or spontaneous mutation of myostatin induced a hypermuscular phenotype in mice and humans $[32,33]$. Therefore, myostatin inactivation may prevent muscle loss in muscular atrophy.

In order to find the muscle protecting potential of 4-HD, we used three kinds of in vitro models to mimic muscle atrophy. In these models, we observed retardation of myogenesis and loss of myotubes together with the reduction of MHC and the increment of myostatin and E3 ligase expressions. It was shown that 4-HD reversed these atrophic conditions by showing increased MHC and decreased myostatin and E3 ligase. The anti-inflammatory potential of 4-HD might have alleviated CM-induced inflammatory environment to stimulate myogenesis [28].

Previous studies have shown that glucocorticoids can induce muscle atrophy through physiological muscle changes in rodents [5,6]. Glucocorticoids can induce catabolic muscle atrophy accompanied by characteristic histopathological changes, including loss of cell organelles, decrease of protein content and muscle fiber diameter [34,35]. In the present study, we evaluated protective effects of ethanol extract of AK (EAK) against dexamethasone-induced muscle degradation in animal experiment. Dexamethasone reduced gastrocnemius muscle thickness, muscle fiber diameters, and body weight of rats, while EAK significantly improved these characteristics of muscle atrophy. EAK also blunted the expression of muscle specific E3 ligases (MuRF1 and MAFbx) in the gastrocnemius muscle of dexamethasone treated rats.

\section{Conclusions}

In summary, the ethanol extract and its active principle, 4-hydroxyderricin from Ashitaba, can overcome the muscle atrophy through double mechanisms of decreasing muscle protein degradation 
and activating myoblast differentiation. It was shown that 4-hydroxyderricin alleviates muscle atrophy by stimulating myogenesis via p38 MAPK activation and protecting muscle waste via down-regulation of E3 ligases (MuRF1 and MAFbx) and myostatin. In muscle atrophic rat model, ethanol extracts of Angelica keiskei Koidzumi protected the loss of body weight and gastrocnemius muscle mass through suppressing the expression of muscle-specific E3 ligases. Taken together, ethanol extract of A. keiskei and 4-hydroxyderricin have therapeutic potential for treating muscle atrophy.

Supplementary Materials: The following are available online at http://www.mdpi.com/2072-6643/11/10/2419/s1, Figure S1: Effect of ethanol extract of Angelica keiskei (EAK) on exercise endurance in dexamethasone-induced muscle atrophied mice.

Author Contributions: Investigation, M.K., H.L., and C.P.; writing-original draft preparation, H.L.; writing-review and editing, H.L., Y.H.C., and J.-H.R.; supervision, Y.H.C., J.-H.R.

Funding: This research was supported by Sookmyung Women's University Specialization Program Funding (No. 1-201809-6). The funding body had no role other than financial support.

Conflicts of Interest: The authors declare no conflict of interest.

\section{References}

1. Dutt, V.; Gupta, S.; Dabur, R.; Injeti, E.; Mittal, A. Skeletal muscle atrophy: Potential therapeutic agents and their mechanisms of action. Pharm. Res. 2015, 99, 86-100. [CrossRef] [PubMed]

2. Guan, M.; Shinde, A.M.; Hendifar, A.E. Pancreatic cancer cachexia: Current concepts and clinical management. In Frailty and Sarcopenia: Onset, Development and Clinical Challenges; IntechOpen: London, UK, 2017; Volume 133.

3. Cruz-Jentoft, A.J.; Landi, F.; Topinkova, E.; Michel, J.P. Understanding sarcopenia as a geriatric syndrome. Curr. Opin. Clin. Nutr. Metab. Care 2010, 13, 1-7. [CrossRef] [PubMed]

4. Lee, M.K.; Kim, Y.M.; Kim, I.H.; Choi, Y.H.; Nam, T.J. Pyropia yezoensis peptide PYP15 protects against dexamethasone induced muscle atrophy through the downregulation of atrogin1/MAFbx and MuRF1 in mouse C2C12 myotubes. Mol. Med. Rep. 2017, 15, 3507-3514. [CrossRef] [PubMed]

5. Clarke, B.A.; Drujan, D.; Willis, M.S.; Murphy, L.O.; Corpina, R.A.; Burova, E.; Rakhilin, S.V.; Stitt, T.N.; Patterson, C.; Latres, E.; et al. The E3 Ligase MuRF1 degrades myosin heavy chain protein in dexamethasone-treated skeletal muscle. Cell Metab. 2007, 6, 376-385. [CrossRef] [PubMed]

6. Lagirand-Cantaloube, J.; Cornille, K.; Csibi, A.; Batonnet-Pichon, S.; Leibovitch, M.P.; Leibovitch, S.A. Inhibition of atrogin-1/MAFbx mediated MyoD proteolysis prevents skeletal muscle atrophy in vivo. PLoS ONE 2009, 4, e4973. [CrossRef] [PubMed]

7. Huang, Y.; Chen, K.; Ren, Q.; Yi, L.; Zhu, J.; Zhang, Q.; Mi, M. Dihydromyricetin Attenuates Dexamethasone-Induced Muscle Atrophy by Improving Mitochondrial Function via the PGC-1alpha Pathway. Cell. Physiol. Biochem. 2018, 49, 758-779. [CrossRef] [PubMed]

8. Caesar, L.K.; Cech, N.B. A Review of the Medicinal Uses and Pharmacology of Ashitaba. Planta Med. 2016, 82, 1236-1245. [CrossRef] [PubMed]

9. Toyama, K.C.S. Ashitaba-Hachijojima Islands Reiso in the Early Modern Period. Kobe Health Welf. Univ. Anal. 2014, 15, 37-44.

10. Chang, H.R.; Lee, H.J.; Ryu, J.H. Chalcones from Angelica keiskei attenuate the inflammatory responses by suppressing nuclear translocation of NF-kappaB. J. Med. Food 2014, 17, 1306-1313. [CrossRef] [PubMed]

11. Zhang, T.; Sawada, K.; Yamamoto, N.; Ashida, H. 4-Hydroxyderricin and xanthoangelol from Ashitaba (Angelica keiskei) suppress differentiation of preadiopocytes to adipocytes via AMPK and MAPK pathways. Mol. Nutr. Food Res. 2013, 57, 1729-1740. [CrossRef] [PubMed]

12. Oh, H.A.; Lee, H.; Park, S.Y.; Lim, Y.; Kwon, O.; Kim, J.Y.; Kim, D.; Jung, B.H. Analysis of plasma metabolic profiling and evaluation of the effect of the intake of Angelica keiskei using metabolomics and lipidomics. J. Ethnopharmacol. 2019, 243, 112058. [CrossRef] [PubMed]

13. Maronpot, R.R. Toxicological assessment of Ashitaba Chalcone. Food Chem. Toxicol. 2015, 77, 111-119. [CrossRef] [PubMed]

14. Nakamura, T.; Tokushima, T.; Kawabata, K.; Yamamoto, N.; Miyamoto, M.; Ashida, H. Absorption and metabolism of 4-hydroxyderricin and xanthoangelol after oral administration of Angelica keiskei (Ashitaba) extract in mice. Arch. Biochem. Biophys. 2012, 521, 71-76. [CrossRef] [PubMed] 
15. Kawabata, K.; Sawada, K.; Ikeda, K.; Fukuda, I.; Kawasaki, K.; Yamamoto, N.; Ashida, H. Prenylated chalcones 4-hydroxyderricin and xanthoangelol stimulate glucose uptake in skeletal muscle cells by inducing GLUT4 translocation. Mol. Nutr. Food Res. 2011, 55, 467-475. [CrossRef] [PubMed]

16. McPherron, A.C.; Guo, T.; Bond, N.D.; Gavrilova, O. Increasing muscle mass to improve metabolism. Adipocyte 2013, 2, 92-98. [CrossRef] [PubMed]

17. Kim, J.W.; Ku, S.K.; Han, M.H.; Kim, K.Y.; Kim, S.G.; Kim, G.Y.; Hwang, H.J.; Kim, B.W.; Kim, C.M.; Choi, Y.H. The administration of Fructus Schisandrae attenuates dexamethasone-induced muscle atrophy in mice. Int. J. Mol. Med. 2015, 36, 29-42. [CrossRef]

18. Baba, K.; Nakata, K.; Taniguchi, M.; Kido, T.; Kozawa, M. Chalcones from Angelica keiskei. Phytochemistry 1990, 29, 3907-3910. [CrossRef]

19. Lee, H.; Lee, S.J.; Bae, G.U.; Baek, N.I.; Ryu, J.H. Canadine from Corydalis turtschaninovii Stimulates Myoblast Differentiation and Protects against Myotube Atrophy. Int. J. Mol. Sci. 2017, 18, 2748. [CrossRef]

20. Zhang, Q.; Shi, X.E.; Song, C.; Sun, S.; Yang, G.; Li, X. BAMBI Promotes C2C12 Myogenic Differentiation by Enhancing Wnt/beta-Catenin Signaling. Int. J. Mol. Sci. 2015, 16, 17734-17745. [CrossRef] [PubMed]

21. Zheng, W.; Hemker, M.L.; Xie, M.; Soukup, S.T.; Diel, P. Anabolic Activity of a Soy Extract and Three Major Isoflavones in C2C12 Myotubes. Planta Med. 2018, 84, 1022-1029. [CrossRef]

22. Kim, D.W.; Curtis-Long, M.J.; Yuk, H.J.; Wang, Y.; Song, Y.H.; Jeong, S.H.; Park, K.H. Quantitative analysis of phenolic metabolites from different parts of Angelica keiskei by HPLC-ESI MS/MS and their xanthine oxidase inhibition. Food Chem. 2014, 153, 20-27. [CrossRef] [PubMed]

23. Oeztuerk-Winder, F.; Ventura, J.J. The many faces of p38 mitogen-activated protein kinase in progenitor/stem cell differentiation. Biochem. J. 2012, 445, 1-10. [CrossRef] [PubMed]

24. Son, Y.H.; Jang, E.J.; Kim, Y.W.; Lee, J.H. Sulforaphane prevents dexamethasone-induced muscle atrophy via regulation of the Akt/Foxo1 axis in C2C12 myotubes. Biomed. Pharm. 2017, 95, 1486-1492. [CrossRef]

25. Ohkura, N.; Atsumi, G.I.; Uehara, S.; Ohta, M.; Taniguchi, M. Ashitaba (Angelica keiskei) Exerts Possible Beneficial Effects on Metabolic Syndrome. OBM Integr. Complement. Med. 2018, 4. [CrossRef]

26. Dedieu, S.; Mazeres, G.; Cottin, P.; Brustis, J.J. Involvement of myogenic regulator factors during fusion in the cell line C2C12. Int. J. Dev. Biol. 2002, 46, 235-241.

27. Ferri, P.; Barbieri, E.; Burattini, S.; Guescini, M.; D’Emilio, A.; Biagiotti, L.; Del Grande, P.; De Luca, A.; Stocchi, V.; Falcieri, E. Expression and subcellular localization of myogenic regulatory factors during the differentiation of skeletal muscle C2C12 myoblasts. J. Cell Biochem. 2009, 108, 1302-1317. [CrossRef]

28. Yasuda, M.; Kawabata, K.; Miyashita, M.; Okumura, M.; Yamamoto, N.; Takahashi, M.; Ashida, H.; Ohigashi, H. Inhibitory effects of 4-hydroxyderricin and xanthoangelol on lipopolysaccharide-induced inflammatory responses in RAW264 macrophages. J. Agric. Food Chem. 2014, 62, 462-467. [CrossRef]

29. Lassar, A.B. The p38 MAPK family, a pushmi-pullyu of skeletal muscle differentiation. J. Cell Biol. 2009, 187, $941-943$. [CrossRef]

30. Jeong, J.; Park, C.H.; Kim, I.; Kim, Y.H.; Yoon, J.M.; Kim, K.S.; Kim, J.B. Korean mistletoe (Viscum album coloratum) extract regulates gene expression related to muscle atrophy and muscle hypertrophy. BMC Complement. Altern. Med. 2017, 17, 68. [CrossRef]

31. Bodine, S.C.; Latres, E.; Baumhueter, S.; Lai, V.K.; Nunez, L.; Clarke, B.A.; Poueymirou, W.T.; Panaro, F.J.; $\mathrm{Na}$, E.; Dharmarajan, K.; et al. Identification of ubiquitin ligases required for skeletal muscle atrophy. Science 2001, 294, 1704-1708. [CrossRef]

32. McPherron, A.C.; Lawler, A.M.; Lee, S.J. Regulation of skeletal muscle mass in mice by a new TGF-beta superfamily member. Nature 1997, 387, 83-90. [CrossRef]

33. Grobet, L.; Pirottin, D.; Farnir, F.; Poncelet, D.; Royo, L.J.; Brouwers, B.; Christians, E.; Desmecht, D.; Coignoul, F.; Kahn, R.; et al. Modulating skeletal muscle mass by postnatal, muscle-specific inactivation of the myostatin gene. Genesis 2003, 35, 227-238. [CrossRef] [PubMed] 
34. Alamdari, N.; Toraldo, G.; Aversa, Z.; Smith, I.; Castillero, E.; Renaud, G.; Qaisar, R.; Larsson, L.; Jasuja, R.; Hasselgren, P.O. Loss of muscle strength during sepsis is in part regulated by glucocorticoids and is associated with reduced muscle fiber stiffness. Am. J. Physiol. Regul. Integr. Comp. Physiol. 2012, 303, R1090-R1099. [CrossRef] [PubMed]

35. Qin, J.; Du, R.; Yang, Y.Q.; Zhang, H.Q.; Li, Q.; Liu, L.; Guan, H.; Hou, J.; An, X.R. Dexamethasone-induced skeletal muscle atrophy was associated with upregulation of myostatin promoter activity. Res. Vet. Sci. 2013, 94, 84-89. [CrossRef] [PubMed]

(C) 2019 by the authors. Licensee MDPI, Basel, Switzerland. This article is an open access article distributed under the terms and conditions of the Creative Commons Attribution (CC BY) license (http://creativecommons.org/licenses/by/4.0/). 